\title{
Produtividade de trigo e soja em Latossolo Vermelho distrófico sob erosão simulada e causada pela chuva(1)
}

\author{
Carlos Gaertner ${ }^{(2)}$, Renato Antonio Dedecek ${ }^{(3)}$ e Rui Maranhão Biscaia(4)
}

\begin{abstract}
Resumo - O objetivo deste trabalho foi avaliar o efeito da erosão hídrica, simulada pela retirada de camadas superficiais de solo e causada pela chuva, na produtividade do trigo e da soja. As espessuras das camadas de solo retiradas foram de $0 \mathrm{~cm}, 3 \mathrm{~cm}, 6 \mathrm{~cm}$ e $14 \mathrm{~cm}$, representando a perda de todo o horizonte Ap. Foram avaliados atributos químicos e físicos do solo até a profundidade de $30 \mathrm{~cm}$. A produtividade do trigo não permitiu estabelecer qualquer correlação com a remoção artificial de camadas de solo, enquanto a da soja foi reduzida em $148 \mathrm{~kg} \mathrm{ha}^{-1}$ por $\mathrm{cm}$ de solo removido na espessura de solo de 0-6 cm e de $105 \mathrm{~kg} \mathrm{ha}^{-1}$ por $\mathrm{cm}$ de solo removido na espessura de 0-14 cm. O teor de $\mathrm{Ca}+\mathrm{Mg}$ e a capacidade de troca de cátions, em diferentes profundidades, foram os atributos do solo que mais influenciaram na produtividade da soja. O teor de $\mathrm{C}$ orgânico foi o único atributo do solo que se correlacionou com as produções de trigo e soja, tanto nas condições de erosão causada pela chuva como nas de simulação de erosão. A simulação do efeito da erosão pela retirada artificial de camadas do solo pode ser utilizada para predizer o efeito da erosão hídrica na produtividade da soja em Latossolo Vermelho, uma vez que a diferença do efeito da remoção artificial no rendimento desta cultura em relação às condições sob chuva foi menor do que $6 \%$.
\end{abstract}

Termos para indexação: Triticum aestivum, Glycine max, propriedade físico-química do solo, solo erodido.

Wheat and soybean productivity on a Dark Red Latosol due to simulated and rainfall erosion

\begin{abstract}
The objective of this work was to evaluate the effect of erosion simulated by removal of surface soil layers and the effect of rainfall erosion on wheat and soybean productivity. Thickness of soil layers removed artificially to simulate erosion effects were $0 \mathrm{~cm}, 3 \mathrm{~cm}, 6 \mathrm{~cm}$ and $14 \mathrm{~cm}$, representing the entire Ap horizon. Wheat productivity did not allow to establish any correlation with thickness of removed soil. The reduction of soybean productivity was $148 \mathrm{~kg} \mathrm{ha}^{-1}$ and $105 \mathrm{~kg} \mathrm{ha}^{-1}$ per cm of soil removed, in 0 to $6 \mathrm{~cm}$ and 0 to $14 \mathrm{~cm}$ layers, respectively. Cation exchange capacity and $\mathrm{Ca}+\mathrm{Mg}$ content, in different soil depth, were the soil attributes that most influenced soybean productivity. Organic carbon content was the only soil attribute to correlate with soybean and wheat yields, in both, rainfall and simulated erosion conditions. Simulation of erosion effect on soybean yield by removal of different soil surface layers can be used, once the difference on soybean productivity compared to the same soil layer lost by natural erosion was less than $6 \%$.
\end{abstract}

Index terms: Triticum aestivum, Glycine max, soil chemicophysical properties, eroded soil.

(1) Aceito para publicação em 10 de setembro de 2003. Extraído da dissertação de mestrado do primeiro autor apresentada à Universidade Federal do Paraná, Curitiba, PR.

(2)Escola Técnica, Caixa Postal 441, CEP 89161-000 Rio do Sul, SC. E-mail: carlgaertner@zipmail.com.br

${ }^{(3)}$ Embrapa-Centro Nacional de Pesquisa de Floresta, Caixa Postal 319, CEP 83411-000 Colombo, PR. E-mail: dedecek@cnpf.embrapa.br

(4)Instituto Agronômico do Paraná, Pólo Curitiba, Caixa Postal 2031, CEP 80011-970 Curitiba, PR. E-mail: nbiscaia@iapar.br

\section{Introdução}

Alguns autores enfatizam que a redução na capacidade de suprimento de água para as plantas é a principal causa da queda de capacidade produtiva em solos erodidos (National Soil Erosion, 1981; Frye et al., 1982; Verity \& Anderson, 1990). A exposição do horizonte B textural traz grandes prejuízos ao rendimento das culturas, por apresentar atributos químicos e físicos desfavoráveis ao crescimento das 
plantas (Lowery et al., 1995). Young (1981) enfatiza que a perda da matéria orgânica do solo pela erosão hídrica reduz a taxa de infiltração da água da chuva no solo e a capacidade de armazenamento de água disponível para as plantas. Verity \& Anderson (1990) observaram menores produtividades de grãos em solo erodido e adubado, apontando o menor teor de matéria orgânica encontrado no solo erodido como o principal fator na redução da qualidade do solo.

Muitos trabalhos avaliam o efeito da erosão sobre a produtividade de cultivos anuais e sobre os atributos químicos e físico-hídricos do solo, retirando camadas superficiais do solo para simular a erosão (Frye et al, 1982; Dedecek, 1987; Spavorek et al., 1991). Segundo Stocking (1984), a interpretação dessas perdas deve ser cautelosa, pois as perdas proporcionais de nutrientes são subestimadas e o efeito sobre as produções pode ser menor do que se semelhantes quantidades de erosão ocorressem naturalmente. Segundo Lal (1988), a erosão natural é seletiva e retira as partículas mais finas e férteis, e a magnitude do efeito da erosão na produtividade depende da concentração de nutrientes no solo; em solos de fertilidade restrita à superfície, o efeito da remoção é mais drástico.

A retirada artificial de uma camada superficial do solo subestima o efeito da erosão hídrica no declínio da produtividade em até 10 vezes (Stocking, 1984), o que significa que, ao remover artificialmente, por exemplo, $10 \mathrm{Mg} \mathrm{ha}^{-1}$ de solo, reproduz-se o efeito negativo na produtividade ocasionado pela remoção de $1 \mathrm{Mg} \mathrm{ha}^{-1}$ por erosão sob chuva natural. Segundo Lal (1988), a taxa de enriquecimento dos sedimentos erodidos por chuva natural segue uma proporção de 3:5 para matéria orgânica, argila e micronutrientes, enquanto segundo Schick et al. (2000), essa relação é maior do que a unidade para C orgânico e nutrientes. Lal (1984) concluiu que, usando chuva simulada, o declínio na produtividade de um Alfisol, na Nigéria, foi cinco vezes menor do que com a retirada artificial da camada superficial do solo.

Comparando a produtividade do milho obtida em condições de erosão artificial pela retirada de camadas superficiais do solo e a obtida em condições de erosão por chuva natural, Lal (1988) observou que o efeito desta foi 16,3 vezes mais drástico no mesmo solo e local. Solos com subsolo fértil são mais está- veis, apresentando menos perdas por erosão (Pierce et al., 1984). Spavorek et al. (1991), em estudo com remoção artificial de camadas superficiais num Argissolo Vermelho latossólico endoálico, observaram queda no rendimento de grãos de milho. Dedecek (1987), trabalhando com Latossolo Vermelho textura argilosa em experimento de erosão simulada pela retirada artificial de camadas superficiais do solo, observou que as reduções dos teores de $\mathrm{Ca}, \mathrm{Mg}, \mathrm{Al}$ e $\mathrm{C}$ orgânico foram as que mais afetaram o rendimento da soja.

O objetivo deste trabalho foi comparar o efeito da erosão simulada pela retirada artificial de camadas superficiais de solo e o efeito da erosão sob chuva natural, na produtividade do trigo e da soja.

\section{Material e Métodos}

O trabalho foi realizado no campo experimental do Pólo Regional do Iapar, em Ponta Grossa, PR, onde, por 17 anos (1977-1994), foi conduzido experimento de quantificação de perdas de solo e água sob chuva natural, sob diferentes sistemas de cultivo. O solo foi caracterizado como Latossolo Vermelho distrófico, horizonte A proeminente, textura muito argilosa e declive de $0,06 \mathrm{~m} \mathrm{~m}^{-1}$. Este solo apresentou na camada de $0-14 \mathrm{~cm}$ as seguintes características: $60 \mathrm{~g} \mathrm{~kg}^{-1}$ de areia, $300 \mathrm{~g} \mathrm{~kg}^{-1}$ de silte, $640 \mathrm{~g} \mathrm{~kg}^{-1}$ de argila, $2,53 \mathrm{Mg} \mathrm{m}^{-3}$ de densidade de partículas, $1,08 \mathrm{Mg} \mathrm{m}^{-3}$ de densidade de solo, $\mathrm{pH}\left(\mathrm{CaCl}_{2}\right), 4,0 ; \mathrm{Al}^{3+}, 2,4 \mathrm{cmol}_{\mathrm{c}} \mathrm{dm}^{-3}$; $\mathrm{Ca}^{2+}+\mathrm{Mg}^{2+}, 1,7 \mathrm{cmol}_{\mathrm{c}} \mathrm{dm}^{-3} ; \mathrm{K}^{+}, 0,13 \mathrm{cmol}_{\mathrm{c}} \mathrm{dm}^{-3} ; \mathrm{P}$, $4,0 \mathrm{mg} \mathrm{dm}^{-3}$; Carbono orgânico, 27,2 $\mathrm{g} \mathrm{kg}^{-1}$. O clima da região é $\mathrm{Cfb}$, segundo Köppen. Os tratamentos de manejo sob chuva tiveram perdas de solo monitoradas, que variaram de $0,05 \mathrm{~cm}$ da camada superficial para o sistema de plantio direto, de $3 \mathrm{~cm}$ para o tratamento solo descoberto em $11 \mathrm{~m}$ de comprimento e de $6 \mathrm{~cm}$ para o tratamento solo descoberto em $22 \mathrm{~m}$ de comprimento.

Ao lado da área experimental sob chuva, retiraram-se da camada superficial do solo, espessuras semelhantes às retiradas pela erosão sob chuva, isto é, $0 \mathrm{~cm}, 3 \mathrm{~cm}, 6 \mathrm{~cm}$ e $14 \mathrm{~cm}$. A remoção de uma camada de $14 \mathrm{~cm}$ do solo foi planejada para simular a perda de todo o horizonte Ap do solo.

O delineamento experimental foi em faixas com quatro repetições para as variáveis de produtividade de trigo e soja, em parcelas de 3,5x3,5 m, com três repetições para as amostragens do solo.

O preparo do solo e a semeadura das culturas foram realizados em nível após a remoção das camadas do solo. Antes do preparo do solo aplicaram-se, a lanço, $2 \mathrm{t} \mathrm{ha}^{-1} \mathrm{de}$ 
calcário dolomítico (PRNT 100\%), metade incorporada até a profundidade de $20 \mathrm{~cm}$, com arado, e a outra metade, até a profundidade de $10 \mathrm{~cm}$, com grade de discos.

O trigo, cultivar IAPAR 41, foi semeado no espaçamento de $0,2 \mathrm{~m}$ entre linhas, com 70 sementes por metro linear, em junho de 1995. A adubação no sulco de semeadura constou de $12 \mathrm{~kg} \mathrm{ha}^{-1} \mathrm{de} \mathrm{N}, 90 \mathrm{~kg} \mathrm{ha}^{-1} \mathrm{de}_{2} \mathrm{P}_{5}$ e $30 \mathrm{~kg} \mathrm{ha}^{-1}$ de $\mathrm{K}_{2} \mathrm{O}$. Aos 40 dias da semeadura, aplicaramse, em cobertura, $60 \mathrm{~kg} \mathrm{ha}^{-1}$ de uréia. A soja, cultivar BR 38 , foi semeada no espaçamento de $0,5 \mathrm{~m}$ entre linhas, com 30 sementes por metro linear, em novembro de 1995, aplicando-se $60 \mathrm{~kg} \mathrm{ha}^{-1}$ de $\mathrm{P}_{2} \mathrm{O}_{5}$ e $\mathrm{K}_{2} \mathrm{O}$, no sulco de semeadura. As colheitas e as trilhas foram manuais. Pesaram-se, além dos grãos, a palha produzida, obtendo-se ainda peso hectolítrico do trigo. A relação grão/palha foi obtida dividindo-se a produção de grãos pela de palha. No trigo, o corte da planta foi rente ao solo em área de $9 \mathrm{~m}^{2}(3 \times 3 \mathrm{~m})$ por parcela e na soja, retirou-se a planta inteira.

Nas determinações da condutividade hidráulica saturada, curva de retenção de água no solo, densidades do solo e de partículas e porosidade, coletaram-se amostras indeformadas de solo em anéis volumétricos, e, nas análises granulométricas e químicas, amostras deformadas, nas camadas de 0-10, 10-20 e 20-30 cm, no pleno florescimento do trigo. As análises químicas, granulométricas e físicohídricas foram realizadas conforme Embrapa (1997).

Nas amostragens de raízes, realizadas no pleno florescimento das culturas, em três locais, por profundida-

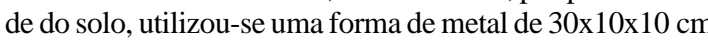
no caso do trigo. Na soja, três amostras foram retiradas com trado tipo holandês, em cada camada de 0-10, 10-20 e 20-30 cm, determinando-se a massa da matéria seca de raízes segundo Bohm (1979). A altura das plantas de trigo foi determinada no pleno florescimento. Na soja efetuaram-se duas determinações: a primeira aos 65 dias e a segunda aos 95 dias após a semeadura. Simultaneamente, realizou-se a contagem do número de plantas por metro linear.

\section{Resultados e Discussão}

O tratamento com remoção de $3 \mathrm{~cm}$ da camada superficial do solo apresentou maior altura média de plantas de trigo do que o sem remoção e com remoção de $6 \mathrm{~cm}$ do solo (Tabela 1). A produtividade do trigo, tanto em grãos como em palha, não apresentou diferença nos diversos tratamentos estudados. Unger et al. (1990) também não encontraram diferenças de produção de trigo, comparando áreas sem remoção e com remoção de $10 \mathrm{~cm}$ de solo, em avaliações realizadas por cinco anos. Já Gaertner (1999) encontrou uma produtividade de trigo nos tratamentos com erosão simulada pela remoção de camadas do solo, aproximadamente $50 \%$ inferior à apresentada pelos tratamentos erodidos por chuva.

Não foi possível estabelecer uma relação entre a produtividade de trigo e a espessura de camada do solo removida artificialmente. Acredita-se que as baixas produtividades decorrentes do ataque observado de doenças fúngicas, principalmente o mal-dopé (Geaumannomyces graminis), tenha contribuído para isto. A diferença de produtividade do trigo entre o tratamento sem remoção do solo e o que teve os $14 \mathrm{~cm}$ superficiais removidos foi de $139 \mathrm{~kg} \mathrm{ha}^{-1}$, o que resultou numa redução média da produtividade de $10 \mathrm{~kg} \mathrm{ha}^{-1}$ por cm de solo removido. Wetter (1977) observou redução de $54 \mathrm{~kg} \mathrm{ha}^{-1}$ de trigo por centímetro de solo removido e encontrou resposta linear negativa entre a produtividade do trigo e a espessura de solo removido.

O tratamento com remoção de $14 \mathrm{~cm}$ da camada superficial do solo apresentou a maior matéria seca de raízes, em todas as profundidades amostradas, mas principalmente na camada de $0-10 \mathrm{~cm}$ de profundidade, onde a matéria seca de raízes foi $45 \%$ maior do que no tratamento sem remoção do solo (Figura 1). É provável que a cultura tenha aumentado a massa de raízes para explorar um volume maior de solo e, com isso, compensar a fertilidade reduzida do tratamento em virtude da remoção total do horizonte Ap. Tal capacidade de adaptação do trigo pode ter sido fator importante para a falta de resposta da produtividade de grãos às diferentes camadas de solo removidas.

A influência da retirada artificial de camadas do solo

Tabela 1. Altura de plantas no pleno florescimento, produção de grãos e matéria seca total do trigo em relação à remoção artificial de camadas do Latossolo Vermelho distrófico ${ }^{(1)}$.

\begin{tabular}{ccccc}
\hline $\begin{array}{l}\text { Camada de solo } \\
\text { removida }(\mathrm{cm})\end{array}$ & $\begin{array}{c}\text { Altura } \\
(\mathrm{cm})\end{array}$ & Grãos & $\begin{array}{c}\text { Matéria } \\
\text { seca }\end{array}$ & $\begin{array}{c}\text { Relação } \\
\text { grão/palha }\end{array}$ \\
\hline 0 (controle) & $0,69 \mathrm{c}$ & $1.025 \mathrm{a}$ & $3.033 \mathrm{a}$ & 0,34 \\
3 & $0,83 \mathrm{a}$ & $969 \mathrm{a}$ & $3.350 \mathrm{a}$ & 0,29 \\
6 & $0,74 \mathrm{bc}$ & $1.011 \mathrm{a}$ & $3.325 \mathrm{a}$ & 0,30 \\
14 & $0,77 \mathrm{ab}$ & $886 \mathrm{a}$ & $3.117 \mathrm{a}$ & 0,28 \\
\hline
\end{tabular}

${ }^{(1)}$ Médias de quatro repetições seguidas de mesma letra, na mesma coluna, não diferem entre si a $5 \%$ de probabilidade pelo teste de Tukey. 
para simular o efeito da erosão hídrica na produção de grãos de soja pode ser observada na Tabela 2 . A produtividade da soja foi praticamente duas vezes maior no tratamento sem remoção artificial do solo do que no tratamento com retirada de $14 \mathrm{~cm}$, resultando numa perda de aproximadamente $100 \mathrm{~kg} \mathrm{ha}^{-1}$ por $\mathrm{cm}$ do solo removido. Na variável altura de plantas, as diferenças observadas não foram significativas, mostrando que o efeito da perda das camadas superficiais do solo foi maior na produção de grãos. Spavorek et al. (1991), trabalhando com perdas de solo simuladas pela remoção de camadas, também observaram que a remoção de parte do horizonte Ap foi menos influente do que a sua remoção total na produtividade do milho.

Comparando-se a produtividade da soja obtida no tratamento sem remoção de camadas do solo com a dos demais tratamentos, observa-se uma redução

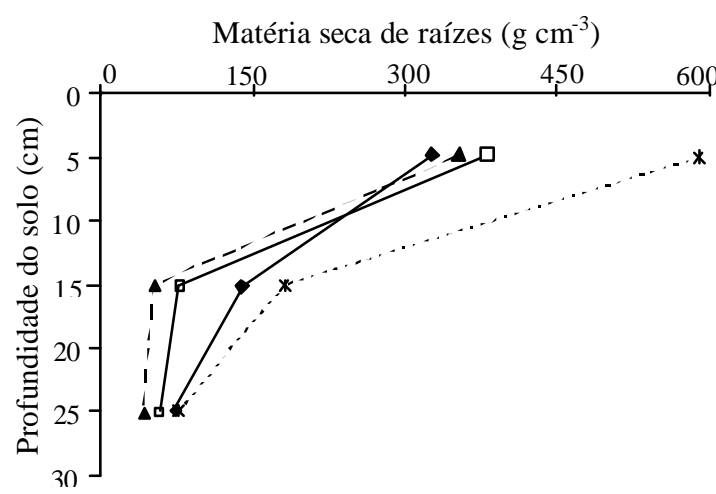

Figura 1. Distribuição da matéria seca de raízes de trigo em relação à profundidade do Latossolo Vermelho distrófico submetido à remoção artificial de camadas de $0 \mathrm{~cm}$ ( controle), $3 \mathrm{~cm}(\square), 6 \mathrm{~cm}(\mathbf{\Delta})$ e $14 \mathrm{~cm}$ (*).

Tabela 2. Altura de plantas no pleno florescimento, produção de grãos e matéria seca total da soja em relação à remoção artificial de camadas do Latossolo Vermelho distrófico ${ }^{(1)}$.

\begin{tabular}{ccccc}
\hline $\begin{array}{l}\text { Camada de solo } \\
\text { removida }(\mathrm{cm})\end{array}$ & $\begin{array}{c}\text { Altura } \\
(\mathrm{cm})\end{array}$ & Grãos & Matéria seca & $\begin{array}{c}\text { Relação } \\
\text { grão/palha }\end{array}$ \\
\hline 0 (controle) & $77,1 \mathrm{a}$ & $3.227 \mathrm{a}$ & $6.056 \mathrm{a}$ & 1,14 \\
3 & $70,9 \mathrm{a}$ & $2.642 \mathrm{ab}$ & $4.922 \mathrm{~b}$ & 1,16 \\
6 & $72,1 \mathrm{a}$ & $2.338 \mathrm{bc}$ & $4.567 \mathrm{~b}$ & 1,05 \\
14 & $70,1 \mathrm{a}$ & $1.750 \mathrm{c}$ & $4.235 \mathrm{~b}$ & 0,70 \\
\hline
\end{tabular}

(1)Médias de quatro repetições seguidas de mesma letra, na mesma coluna não diferem entre si a $5 \%$ de probabilidade pelo teste de Tukey. de $190 \mathrm{~kg} \mathrm{ha}^{-1}$ por $\mathrm{cm}$ de solo perdido, no tratamento de $3 \mathrm{~cm}$ de solo removidos, $148 \mathrm{~kg} \mathrm{ha}^{-1}$ por $\mathrm{cm}$ no de $6 \mathrm{~cm}$ e $105 \mathrm{~kg} \mathrm{ha}^{-1}$ por $\mathrm{cm}$ no tratamento com remoção de todo o horizonte Ap. Assim, a melhor correlação estabelecida entre a produtividade da soja e a remoção artificial de camadas do solo foi uma polinomial de segunda ordem (Figura 2), diferente da obtida por Gaertner (1999) nos tratamentos de perdas de solo por chuva, em que a regressão foi linear. No entanto, na mesma faixa de amplitude de variação da espessura do solo, entre 0 e $6 \mathrm{~cm}$, nas condições de simulação, a regressão também foi linear, com um elevado índice de correlação e uma perda de produtividade média de soja de $148 \mathrm{~kg} \mathrm{ha}^{-1}$ por $\mathrm{cm}$ de solo removido (Figura 2).

Uma vez que a produtividade do trigo não apresentou diferenças significativas em razão das diferentes espessuras de camadas do solo removidas, não se poderia esperar que fossem obtidas correlações significativas da produtividade com os atributos químicos e físicos do solo analisados (Tabela 3). Assim, a variação da produtividade de grãos de trigo foi explicada de forma significativa, embora em menos de $40 \%$, pelo teor de água do solo na saturação (que é a porosidade total determinada) e pelo teor de C orgânico do solo.

Já a produtividade da soja apresentou correlações significativas e com maior número de atributos químicos e físicos do solo, com coeficiente de deter-

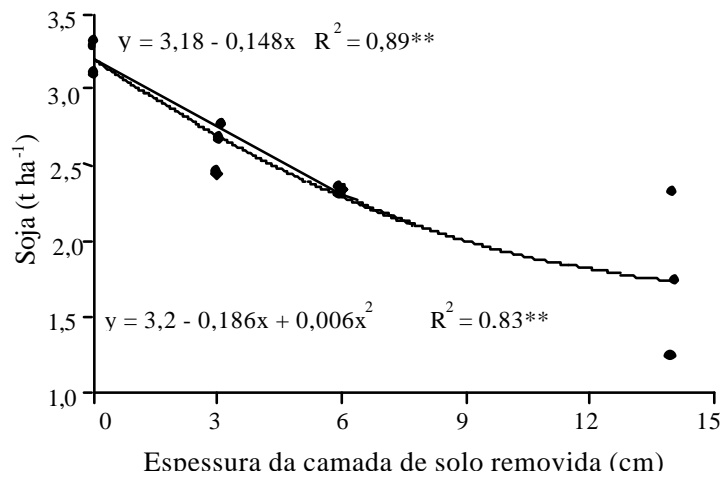

Figura 2. Relação entre a produtividade da soja e a espessura das camadas do Latossolo Vermelho distrófico removidas artificialmente. Regressão polinomial de $2^{\mathfrak{a}}$ ordem relativa aos quatro níveis de erosão simulada e regressão linear relativa aos três primeiros níveis de erosão simulada. **Significativo a $1 \%$ de probabilidade pelo teste $\mathrm{F}$. 
minação acima de 0,5 (Tabela 3). Entre os atributos físicos, a melhor correlação ocorreu entre a produtividade da soja e o teor de água disponível, considerada na faixa entre 6 e $300 \mathrm{kPa}$, concordando com vários autores que enfatizam a redução da capacidade do solo de suprimento de água para as plantas como a principal causa da queda da capacidade produtiva dos solos erodidos (National Soil Erosion, 1981; Frye et al., 1982; Verity \& Anderson, 1990).

$\mathrm{O}$ teor de $\mathrm{Ca}+\mathrm{Mg}$, medido na camada de $0-10 \mathrm{~cm}$, e a CTC, medida na camada de $20-30 \mathrm{~cm}$, foram os atributos químicos que melhor se correlacionaram com a produtividade da soja (Figura 3). À exceção

Tabela 3. Coeficientes de determinação $\left(R^{2}\right)$ entre atributos físicos e químicos do solo, em diferentes camadas do Latosolo Vermelho distrófico, e a produtividade de grãos de soja e de trigo.

\begin{tabular}{lccc}
\hline Atributo & $\begin{array}{c}\text { Profundidade } \\
(\mathrm{cm})\end{array}$ & Soja & Trigo \\
\hline Macroporosidade $^{(1)}$ & $10-20 \mathrm{~cm}$ & $0,38^{*}$ & \\
Porosidade total $_{\text {Teor de água disponível }}^{(2)}$ & $10-20 \mathrm{~cm}$ & & $0,37^{*}$ \\
Ca+Mg & $10-20 \mathrm{~cm}$ & $0,58^{* *}$ & \\
P & $0-10 \mathrm{~cm}$ & $0,68^{* *}$ & \\
C orgânico & $10-20 \mathrm{~cm}$ & $0,50^{* *}$ & \\
K & $20-30 \mathrm{~cm}$ & $0,53^{* *}$ & $0,34^{*}$ \\
CTC & $20-30 \mathrm{~cm}$ & $0,53^{* *}$ & \\
\hline
\end{tabular}

${ }^{(1)}$ Saturação a $6 \mathrm{kPa} .{ }^{(2)}$ Entre 300 e $6 \mathrm{kPa}$ * * **Significativo a $5 \%$ e a $1 \%$ de probabilidade, respectivamente, pelo teste $\mathrm{F}$.

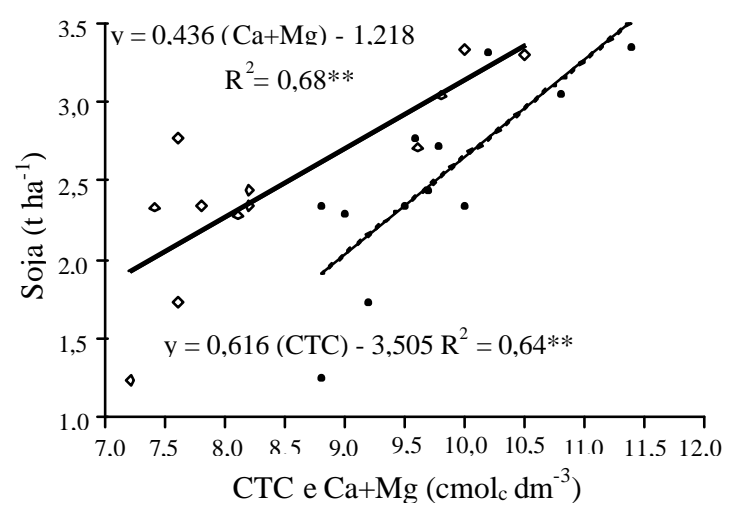

Figura 3. Relações da produtividade da soja com a capacidade de troca de cátions (CTC, $\bullet$ ) na camada de $20-30 \mathrm{~cm}$, e com os teores de $\mathrm{Ca}+\mathrm{Mg}(\diamond)$ na camada de 0-10 cm do Latossolo Vermelho distrófico (média de três repetições). $* *$ Significativo a $1 \%$ de probabilidade pelo teste F. do teor de $\mathrm{Ca}+\mathrm{Mg}$, os demais atributos do solo que apresentaram correlação com a produção de trigo e soja são altamente dependentes do teor de $\mathrm{C}$ orgânico no solo, o qual também apresentou correlação com a produtividade nos dois cultivos. Comparando-se o tratamento mais produtivo, sem remoção artificial de camadas do solo, com aquele mais produtivo, com remoção de todo o horizonte Ap, a diferença no teor de $C$ orgânico foi de $4,7 \mathrm{~g} \mathrm{dm}^{-3}$ nas três profundidades de solo amostradas. Segundo Bruce et al. (1988), as variáveis que mais influenciaram a produtividade da soja foram C orgânico, principalmente nos solos muito erodidos, $\mathrm{pH}$ e tensão de água no solo. Segundo Stocking (1984), a CTC, na camada até $50 \mathrm{~cm}$, e as deficiências de N, P e K foram as variáveis mais influentes na produtividade da soja. Dedecek (1987), trabalhando com Latossolo Vermelho, verificou que as melhores correlações foram estabelecidas entre a produtividade de soja e teores de $\mathrm{Ca}+\mathrm{Mg}$, seguidas da porcentagem de matéria orgânica e teor de Al na camada de $0-40 \mathrm{~cm}$.

No trigo, para uma determinada espessura de camada do solo removida artificialmente, a produtividade foi aproximadamente $30 \%$ menor que a observada para a mesma espessura de camada erodida pela chuva (Tabela 4 ).

As produtividades da soja em solo erodido pela remoção artificial do solo e pela chuva foram semelhantes (Tabela 4). A perda de $3 \mathrm{~cm}$ de solo causou uma diferença de apenas $1,3 \%$ entre a produtividade da soja sob erosão simulada e sob chuva; na espes sura de $6 \mathrm{~cm}$, a diferença de produção foi de $5,6 \%$. De 0-6 cm, as regressões estabelecidas foram lineares com relação à produtividade da soja, com eleva-

Tabela 4. Produtividade de grãos de trigo e soja em Latossolo Vermelho distrófico sob erosão causada por chuva e por remoção artificial de camadas do solo ${ }^{(1)}$.

\begin{tabular}{ccl}
\hline $\begin{array}{c}\text { Camada de solo } \\
\text { removida e perdida }(\mathrm{cm})\end{array}$ & Trigo & \multicolumn{1}{c}{ Soja } \\
\hline $3^{(2)}$ & $1.444 \mathrm{a}$ & $2.610 \mathrm{ab}$ \\
3 & $969 \mathrm{ab}$ & $2.642 \mathrm{a}$ \\
$6^{(2)}$ & $1.385 \mathrm{a}$ & $2.476 \mathrm{ab}$ \\
6 & $1.011 \mathrm{ab}$ & $2.338 \mathrm{ab}$ \\
14 & $886 \mathrm{~b}$ & $1.750 \mathrm{~b}$ \\
\hline $\mathrm{CV}(\%)$ & 20.4 & 15.7 \\
\hline
\end{tabular}

${ }^{(1)}$ Médias de quatro repetições seguidas de mesma letra, na mesma coluna, não diferem entre si a $5 \%$ de probabilidade pelo teste de Tukey. ${ }^{(2)}$ Camada de solo perdida por chuva. 
do coeficiente de correlação. A redução da produtividade foi de $148 \mathrm{~kg} \mathrm{ha}^{-1}$ por $\mathrm{cm}$ de solo removido para a retirada artificial de $6 \mathrm{~cm}$ de solo, enquanto para a perda da mesma camada sob chuva a redução foi de $139 \mathrm{~kg} \mathrm{ha}^{-1}$ por $\mathrm{cm}$ de solo removido. Assim, a perda de produtividade na erosão simulada foi $6 \%$ superior à obtida sob chuva natural, uma diferença muito pequena considerando-se a variabilidade existente em experimentos dessa natureza. Esses dados contrariam os de outros autores (Lal, 1984, 1988; Stocking, 1984), que afirmam que a simulação da erosão pela retirada de camadas de solo é mais drástica do que sob chuva natural. Trabalhando com solo semelhante, Dedecek et al. (1986) observaram que os sedimentos produzidos em chuvas de grande magnitude eram muito semelhantes, em concentração de nutrientes, matéria orgânica e granulometria, à camada superficial do solo que lhe deu origem. Também observaram que esses eventos, em número aproximado de 10 por ano agrícola, eram responsáveis por $80 \%$ das perdas de solo por erosão sob chuva natural (Dedecek et al., 1986; Dedecek, 1988).

Nos tratamentos com retirada artificial de camadas do solo para simular a erosão, o teor de C no solo foi o único atributo, que se correlacionou com a produtividade de ambas as culturas (Tabela 3). Todos os demais atributos do solo apresentaram correlação significativa com a produtividade de cada cultura. O mesmo aconteceu com a erosão causada pela chuva, embora, nesse caso o coeficiente de determinação entre a produtividade da soja e o teor de $\mathrm{C}$ no solo tenha sido menor, bem como o nível de significância. Isso mostra que há uma perda maior de matéria orgânica com a retirada artificial das camadas superficiais do solo do que com a erosão sob chuva, o que pode afetar mais fortemente a redução da produtividade das culturas, mas com menor intensidade do que a literatura relata, pelo menos para o solo deste estudo. Em relação à correlação entre a produtividade do trigo e o teor de $\mathrm{C}$ no solo, os valores do coeficiente de determinação e do nível de significância foram menores do que para a soja.

\section{Conclusões}

1. O trigo apresenta maior capacidade do que a soja em aumentar o volume de raízes em condições adversas, ocasionadas pela remoção artificial de camadas de solo.

2. O teor de $\mathrm{Ca}+\mathrm{Mg}$, na camada de $0-10 \mathrm{~cm}$ do solo, e a capacidade de troca de cátions, na camada de $20-30 \mathrm{~cm}$, mostram as maiores correlações com a produtividade da soja, em diferentes espessuras de camadas superficiais removidas do solo.

3. O teor de C orgânico afeta a produtividade de trigo e soja, nas condições de erosão simulada e erosão causada pela chuva.

4. A redução da produtividade da soja ocasionada pela erosão simulada é semelhante à causada pela perda do solo por chuva, permitindo o uso da remoção artificial de camadas do solo para avaliar o efeito da erosão hídrica na produtividade da soja em Latossolo Vermelho.

\section{Agradecimentos}

À chefia e aos técnicos e pessoal de apoio do Iapar/Pólo Regional de Ponta Grossa, PR, pela contribuição na realização deste trabalho.

\section{Referências}

BOHM, W. Methods of studying root systems. Berlin: Springer, 1979. 189 p.

BRUCE, R. R.; WHITE JUNIOR, A. W.; THOMAS, A. W.; SNYDER, W. M.; LANGDALE, G. W. Characterization of soil-crop yield relations over a range of erosion on a landscape. Geoderma, Amsterdam, v. 43, p. 99-116, 1988.

DEDECEK, R. A. Efeitos das perdas e deposições de camadas de solo na produtividade de um Latossolo Vermelho-Escuro dos Cerrados. Revista Brasileira de Ciência do Solo, Campinas, v. 11, p. 323-328, 1987.

DEDECEK, R. A. Fatores de erosividade da chuva, enxurrada e perdas de solo sob condições de cerrado. Pesquisa Agropecuária Brasileira, Brasília, v. 23, n. 12, p. 14311438, dez. 1988.

DEDECEK, R. A.; RESCK, D. V. S.; FREITAS JUNIOR, E. de. Perdas de solo, água e nutrientes por erosão em latossolo vermelho-escuro dos cerrados em diferentes cultivos sob chuva natural. Revista Brasileira de Ciência do Solo, Campinas, v. 10, p. 265-272, 1986.

EMBRAPA. Centro Nacional de Pesquisa de Solos (Rio de Janeiro, RJ). Manual de métodos de análise de solos. Rio de Janeiro, 1997. 212 p. 
FRYE, W. W.; ABELHAR, S. A.; MURDOCK, L. W.; BLEVINS, R. L. Soil erosion effects on properties and productivity of two Kentucky soils. Soil Science Society of America Journal, Madison, v. 46, p. 1051-1055, 1982.

GAERTNER, C. Erosão natural e simulada e produtividade em latossolo vermelho-escuro. 1999. $97 \mathrm{f}$. Dissertação (Mestrado em Agronomia) - Universidade Federal do Paraná, Curitiba, 1999.

LAL, R. Monitoring soil erosion's impact on crop productivity. In: SOIL AND WATER CONSERVATION SOCIETY (Ankeny, Estados Unidos). Soil erosion research methods. Ankeny, 1988. p. 187-202.

LAL, R. Soil erosion on Alfisols in Western Nigeria - I: the changes in physical properties and response of crops. Geoderma, Amsterdam, v. 16, p. 29-31, 1984.

LOWERY, B.; SHAWN, J.; SCHUMACHER, T.; JONES, A. Physical properties of selected soils by erosion class. Journal of Soil and Water Conservation, Ankeny, v. 50, n. 3, p. 306-311, 1995.

NATIONAL SOIL EROSION. Soil Productivity Research Planning Committee. Soil erosion effects on soil productivity: a research perspective. Journal of Soil and Water Conservation, Ankeny, v. 36, p. 82-90, 1981.

PIERCE, F. J.; DOWDY, R. H.; LARSON, W. E.; GRAHAM, W. A. P. Soil productivity in the corn belt: assessing long-term effects. Journal of Soil and Water Conservation, Ankeny, v. 39, p. 131-136, 1984.
SCHICK, J.; BERTOL, I.; BALBINOT JUNIOR, A. A.; BATISTELA, O. Erosão hídrica em Cambissolo Húmico alumínico submetido a diferentes sistemas de preparo e cultivo do solo - II: perdas de nutrientes e carbono orgânico. Revista Brasileira de Ciência do Solo, Viçosa, MG, v. 24, p. 437-447, 2000.

SPAVOREK, G.; TERAMOTO, E. R.; TORET, D. M.; ROCHELE, T. C. P. Erosão simulada e a produtividade da cultura do milho. Revista Brasileira de Ciência do Solo Campinas, v. 15, p. 363-368, 1991

STOCKING, M. Erosion-induced in soil productivity: a research design. Rome: FAO, 1984. 33 p. (Soil Conservation Programme Consult's Working Paper, 1).

UNGER, P. W.; FULTON, L. J.; JONES, O. R. Landleveling effects on soil texture, organic matter content, and aggregate stability. Journal of Soil and Water Conservation, Ankeny, v. 45, p. 412-415, 1990.

VERITY, G. E.; ANDERSON, D. W. Soil erosion effects on soil quality and yield. Canadian Journal of Soil Science, Ottawa, v. 70, p. 471-484, 1990.

WETTER, F. The influence of topsoil depth on yields . Colfax: United States Department of Agriculture, 1977. 3 p. (Technical Notes AGRON-10).

YOUNG, K. K. The impact of erosion on the productivity of soils in the United States. Washington: J. Wiley, 1981. p. 295-303. 